Departamento de Nutrición, Diabetes y Metabolismo, Escuela de Medicina, Pontificia Universidad Católica de Chile. ${ }^{a}$ Tesista, Programa de Magíster en Nutrición. Pontificia Universidad Católica de Chile.

bBioquímico, Doctor en Ciencias.

Financiamiento: Fondecyt 11100168 (V.A.C.). Conicyt-PAl 79100018 (V.A.C.) Fondecyt 1120586 (J.L.S.). Dirección de Investigación, Escuela de Medicina, Pontificia Universidad Católica de Chile (D.G.)

Recibido el 19 de marzo de 2013, aceptado el 9 de diciembre de 2013.

Correspondencia a: Dr. Víctor A. Cortés Mora Departamento de Nutrición Diabetes y Metabolismo, Pontificia Universidad Católica de Chile.

Marcoleta 367, Santiago, Chile. Teléfono: 56223543862 vcortes@med.puc.cl

\section{Nuevas proyecciones fisiológicas, patológicas y terapéuticas de la leptina}

\author{
DENISSE GOLDENBERG ${ }^{\mathrm{a}}$, JOSÉ LUIS SANTOS ${ }^{\mathrm{b}}$, \\ MARÍA ISABEL HODGSON, VÍCTOR A. CORTÉS
}

\section{Novel physiological and therapeutic implications of leptin}

The adipose tissue is an endocrine organ that produces a variety of protein hormones. One of them is leptin, which regulates several critical functions at the central nervous system such as caloric intake, basal energy expenditure, reproduction, glucose and lipid metabolism and osteogenesis. Acting at a local level, leptin modulates the immune system and promotes liver fibrogenesis. The most promising therapeutic implications of leptin will possibly be in type 1 diabetes mellitus (DM1). Its supplementation in animal models of DM1 prevents hyperglycemia and ketoacidosis. These actions depend on the activation of leptin receptors in the central nervous system and the suppression of glucagon signaling in the liver.

(Rev Med Chile 2014; 142: 738-747)

Key words: Diabetes mellitus; Leptin; Lipodystrophy; Obesity.
L a epidemia mundial de obesidad ha motivado un gran número de investigaciones relativas al tejido adiposo. Entre los descubrimientos más notables de esta área está el de una variedad de factores secretados por el tejido adiposo y con actividad reguladora sobre la ingesta calórica, gasto energético, reproducción, actividad locomotora, metabolismo glucídico y lipídico, respuesta inmune y fisiología ósea (Figura 1A).

Entre estas hormonas adipocitarias, colectivamente denominadas "adipoquinas", destaca la leptina, la primera en ser identificada y la más estudiada hasta la fecha.

Esta proteína fue descubierta en 1994 por Jeffrey Friedman y cols., como resultado de la búsqueda de la anormalidad genética responsable del fenotipo de los ratones $o b / o b^{1}$. Estos animales, conocidos desde $1950^{2}$, presentan un síndrome hereditario autosómico recesivo caracterizado por hiperfagia, obesidad severa, gasto energético disminuido, hipoactividad, resistencia a la insulina, diabetes mellitus, esteatosis hepática e infertilidad. Friedman y cols. determinaron que la causa del fenotipo ob/ob es la mutación de un único nucleótido (sustitución de una citosina por una timidina) en la posición 105 de la secuencia codificante del gen Lep, lo que determina la generación de un codón de detención prematuro e incapacidad para sintetizar leptina ${ }^{3}$.

Se han descrito mutaciones inactivantes en el gen $L E P$ en pacientes con obesidad mórbida precoz $^{4}$. En ellos ${ }^{5}$, así como en pacientes con lipodistrofia generalizada ${ }^{6}$ y en ratones $o b / o b^{7}$, la administración de leptina revierte rápida y dramáticamente todas las anormalidades metabólicas, demostrando el rol causal de la ausencia de esta hormona en estas enfermedades.

Pese al potente efecto terapéutico de la suplementación de leptina en humanos y ratones con carencia de leptina endógena, la eficacia de esta hormona en el tratamiento de la obesidad común ha sido inconsistente ${ }^{8-10}$, posiblemente como consecuencia de grados variables de resistencia a la acción de esta hormona en estos sujetos ${ }^{11}$. 
Leptina en salud y enfermedad - D. Goldenberg et al

En este artículo se revisan algunos aspectos bioquímicos y moleculares de la leptina y sus sistemas de señalización intracelular, y se examinan hallazgos recientes sobre el rol de esta hormona en la inmunidad innata y adquirida, fibrosis hepática, metabolismo óseo, reproducción y diabetes mellitus tipo 1 y tipo 2 (Figura 1B). Las acciones de la leptina sobre la regulación del apetito y el balance energético han sido revisadas en excelentes artículos previos $^{1,12}$.

\section{Aspectos moleculares y mecanismos de acción de la leptina}

La leptina es una hormona peptídica de 167 aminoácidos sintetizada por los adipocitos del tejido adiposo blanco (Figura 2A) ${ }^{3,13}$. Su estructura terciaria es similar a la de citoquinas de cadena larga helicoidal, tales como el factor inhibidor de la leucemia y la interleuquina 6 (Figura $2 \mathrm{~A})^{14}$. Esta hormona es secretada a la circulación y se une a receptores específicos de superficie celular, gatillando la activación de vías específicas de señalización intracelular ${ }^{15}$.

Otros tejidos tales como placenta ${ }^{16}$ y mucosa gástrica ${ }^{17}$ podrían producir leptina en pequeñas cantidades; sin embargo, el significado fisiológico o patológico de estos hallazgos permanece desconocido.

\section{A. Receptores de leptina}

El gen del receptor de leptina (Lepr) fue identificado y clonado en el plexo coroideo y está mutado en los ratones $d b / d b^{15}$. Estos animales son una fenocopia del ratón $o b / o b$, pero se diferencian de ellos en que son completamente insensibles a la infusión de leptina exógena.

Lepr pertenece a la familia de receptores de citoquinas tipo $\mathrm{I}^{15} \mathrm{y}$ codifica seis isoformas distintas del receptor, denominadas alfabéticamente LepRa a LepRf. Estas isoformas se originan por corte y empalme (splicing) alternativo del ARN mensajero original ${ }^{18}$ y comparten un dominio extracelular y otro de transmembrana común. El dominio intracelular es, por el contrario,variable y característico de cada isoforma ${ }^{19}$. Así, existen varias formas cortas (LepRa, LepRc, LepRd y LepRf), una larga (LepRb) y una soluble (LepRe), esta última detectable en la circulación sistémica (Figura 2B) $)^{20}$.
De estas proteínas, sólo LepRb posee los elementos estructurales necesarios para la transducción de señales intracelulares ${ }^{13,19}$. De hecho, el ratón $d b / d b$ originalmente descrito carece exclusivamente de LepRb, estando conservadas las otras variantes de $\operatorname{LepR}^{18,21}$.

LepRb se expresa normalmente en diversos grupos neuronales del sistema nervioso central (SNC), incluyendo los núcleos hipotalámicos arcuato, dorsomedial, paraventricular, ventromedial y lateral ${ }^{13}$, además de algunos núcleos talámicos y cerebelosos $^{22}$. La relevancia funcional de LepR en el SNC ha sido demostrada por medio de su su-

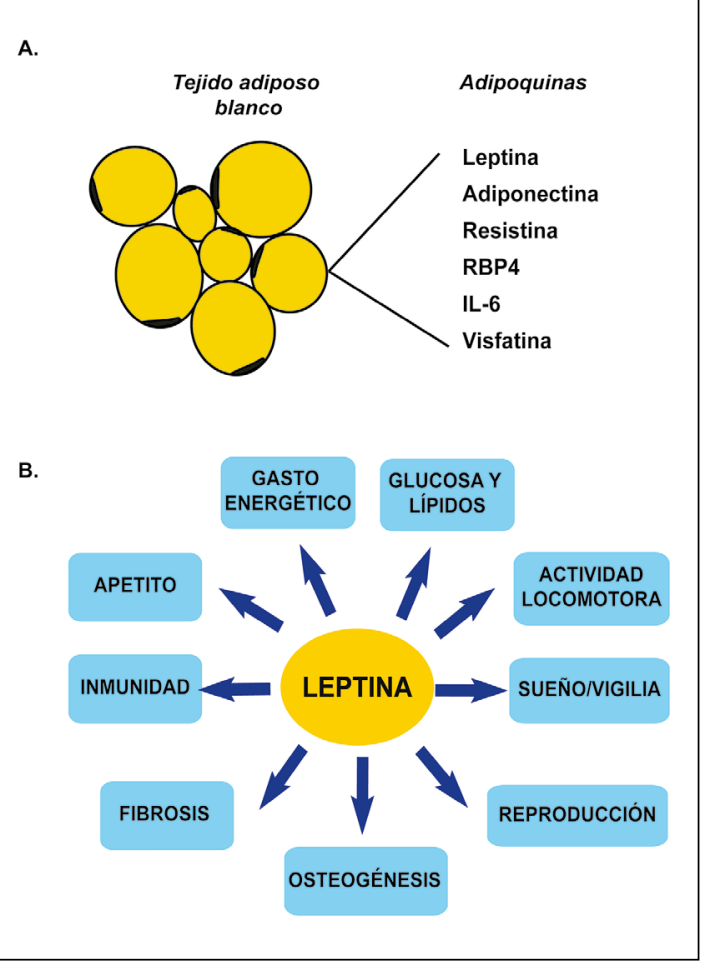

Figura 1. La leptina es una hormona de origen adipocitario con diversas acciones fisiológicas. (A) Las células parenquimatosas del tejido adiposo blanco producen y secretan a la circulación general varias proteínas con acción hormonal (adipoquinas) entre las que destaca la leptina. Muchas adipoquinas tienen efectos reguladores sobre la sensibilidad insulínica, mejorándola (adiponectina, IL-6) o reduciéndola (resistina, RBP4). (B) Se han descrito numerosos efectos fisiológicos de la leptina tanto en modelos experimentales en animales como en pacientes con defectos genéticos primarios de la secreción o acción de esta hormona (ver texto para información detallada). Abreviaturas: IL-6, interleuquina 6 . RBP4, retinol binding protein 4. 
A.

MHWGTLCGFLWLWPYLFYVQAVPIQKVQDDTKTLIKTIVTRINDISHTQSVSSKQ KVTGLDFIPGLHPILTLSKMDQTLAVYQQILTSMPSRNVIQISNDLENLRDLLHVL AFSKSCHLPWASGLETLDSLGGVLEASGYSTEVVALSRLQGSLQDMLWQLDLS PGC

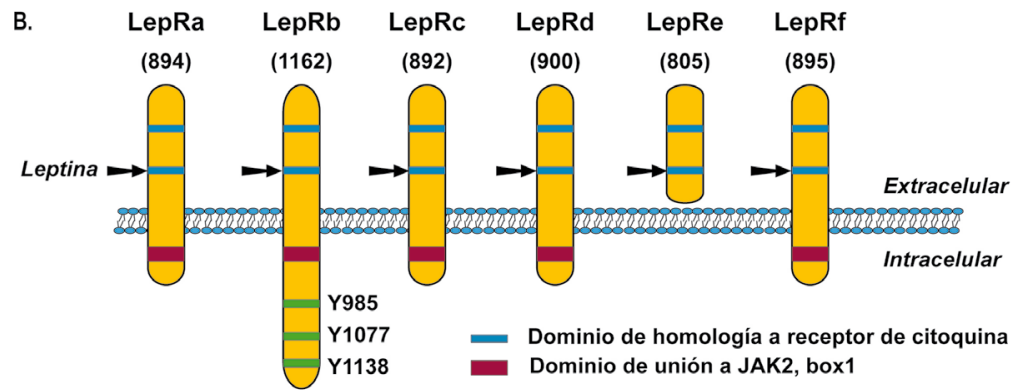

C.

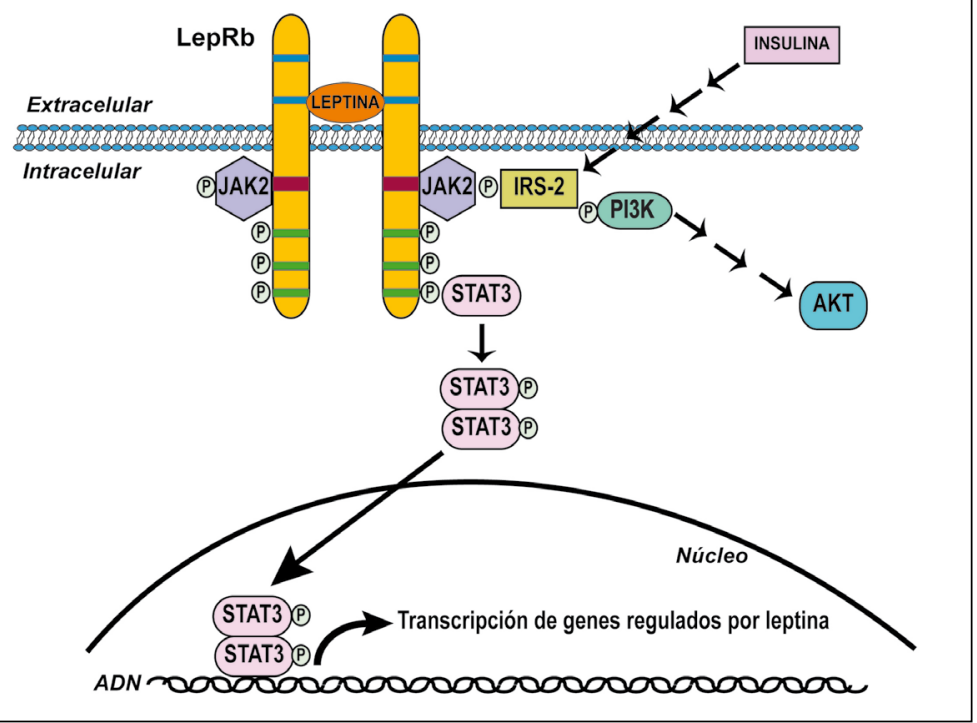

Figura 2. Estructura de la leptina y de sus receptores y mecanismos de señalización intracelular de la leptina. (A) La leptina humana está constituida por 167 aminoácidos, de los cuales los primeros 21 (destacados en amarillo) forman el péptido señal, el cual determina el procesamiento de esta hormona y su posterior secreción a la circulación. La estructura secundaria de la leptina es similar al citoquinas de cadena larga helicoidal, constando de 4 hélices alfa (aminoácidos destacados en celeste). (B) Las distintas isoformas del receptor de leptina son codificadas por un único gen (LEPR) que da origen a 6 isoformas (LepRa a LepRf). Todas ellas tienen distinto número de aminoácidos (señalado entre paréntesis) y comparten los dominios extracelular y transmembrana, La excepción es LepRe, que carece del dominio transmembrana y se encuentra soluble en la circulación. LepRb es la isoforma más larga y es la única que tiene los elementos estructurales necesarios para la activación de cascadas de señalización intracelular, en particular los residuos tirosina (Y) 985, 1077 y 1138, esenciales para la interacción con otras proteínas intracelulares. (C) Como consecuencia de la unión de leptina al segundo dominio de homología a receptor de citoquina del dominio extracelular de LepRb, se induce dimerización en la superficie celular y asociación de JAK2 al dominio intracelular en la región "box 1". Esta asociación induce un cambio conformacional en esta última proteína, potenciando su actividad quinasa de residuos tirosina, resultando en fosforilación de Y985, Y1077, Y1138 y autofosforilación de JAK2, lo que genera nuevos sitios de unión para proteínas intracelulares que transducen señales, tales como STAT3 e IRS-2. STAT3 también es fosforilada por JAK2, tras lo cual forma un homodímero que se trasloca al núcleo celular en donde se une a secuencias nucleotídicas específicas en las regiones reguladoras de los genes controlados por leptina. IRS-2 también es fosforilado por el receptor de insulina, siendo un punto de unión entre las dos vías de señalización. Abreviaturas: JAK2, Janus kinase 2. IRS-2, insulin receptor substrate-2. PI3K, phosphoinositide 3-kinase.STAT3, signal transducer and activator of transcription 3. 
presión génica selectiva en neuronas en ratones ${ }^{23}$. Éstos desarrollan hiperfagia severa, obesidad y diabetes, remedando a los ratones $d b / d b$ y $o b / o b$, a pesar de expresar LepR en todas las otras células del organismo ${ }^{23}$, indicando que las acciones metabólicas de la leptina dependen mayoritaria, si es que no exclusivamente, de la activación de LepRb en el SNC.

A nivel periférico, LepRb se expresa en pulmón, riñón, músculo esquelético, hígado, bazo, corazón, páncreas, corteza adrenal, ovarios, testículos, tejido adiposo y tracto gastrointestinal ${ }^{15,18}$. Sin embargo, dado que la supresión génica selectiva de LepRb en células no neuronales no genera alteraciones metabólicas detectables en ratones, su función permanece desconocida.

Las isoformas cortas de LepR se encuentran en altos niveles en el plexo coroideo y en vasos capilares del SNC, por lo que se especula que pudieran estar involucradas en el movimiento de leptina a través de la barrera hematoencefálica ${ }^{24}$.

La forma soluble de LepR constituye el principal sitio de unión de leptina en la sangre y sus niveles correlacionan inversamente con la adiposidad corporal y la concentración de leptina plasmática $^{25}$. Su función también es desconocida; sin embargo, se ha reportado que los niveles plasmáticos de LepR se correlacionan inversamente con el riesgo de desarrollar diabetes mellitus tipo $2(\mathrm{DM} 2)^{26}$.

\section{B. Señalización intracelular dependiente de $L e p R b$}

El LepRb transmite señales intracelulares por medio de la activación de la vía JAK (janus kinase)/STAT (signal transducer and activator of transcription $)^{15,20}$.

La unión de leptina a LepRb determina la dimerización del receptor y la unión de JAK2 a su dominio citoplasmático. Ahí, JAK2 fosforila residuos tirosina de LepRb y gatilla el reclutamiento de proteínas críticas para la transducción de señales ${ }^{12}$. De estas destaca STAT3, la cual también es fosforilada por JAK2, dimerizándose y translocándose al núcleo celular. En esta ubicación STAT3 se une a sitios específicos del ADN y promueve la transcripción de genes regulados porleptina $^{27}$ (Figura 2C).

JAK2 fosforila y activa IRS-2 (insulin receptor substrate-2), induciendo la activación de PI3K (phosphoinositide 3-kinase). Dado que la trans- ducción de señales dependiente del receptor de insulina (RI) es mediada por PI3K, IRS-2 constituye un punto de unión entre las vías de señalización dependientes de leptina e insulina ${ }^{20}$ (Figura 2C). De manera relevante, el RI se encuentra ampliamente expresado en el sistema nervioso central y podría participar en la regulación del apetito, complementariamente al sistema dependiente de leptina. De hecho, la eliminación neuronal selectiva de RI en ratones, determina mayor ingesta alimentaria y susceptibilidad aumentada a obesidad inducida por $\operatorname{dieta}^{28}$, sin embargo, la relevancia de estas observaciones en humanos permanece desconocida.

\section{Aspectos fisiopatológicos de la leptina}

\section{A. Inmunidad}

Varias líneas de evidencia sugieren que la leptina ejerce roles inmunomoduladores y que alteraciones en las vías de señalización dependientes de esta hormona podrían ser relevantes en enfermedades inflamatorias humanas ${ }^{29}$. Primero, los niveles de leptina aumentan en infecciones agudas ${ }^{30} y$ en respuesta a diversos estímulos proinflamatorios $^{31,32}$; además LepRb se expresa en células del sistema inmune, incluyendo monocito/macrófagos, polimorfonucleares neutrófilos (PMN) y linfocitos ${ }^{33}$. Estudios en cultivos celulares han mostrado que leptina tiene efectos quimioatrayentes y activadores en monocitos/macrófagos ${ }^{34}$ y PMN in vitro ${ }^{35,36}$.

Los ratones $o b / o b$ tienen atrofia tímica y un número reducido de timocitos $\mathrm{CD} 4+\mathrm{y} \mathrm{CD} 8+\mathrm{y}$ de células natural killer en hígado, bazo, pulmones y sangre periférica ${ }^{13,37,38}$. La infusión de leptina normaliza estas variables ${ }^{37,39,40}$. Similarmente, humanos con deficiencia genética de leptina tienen respuestas Th1 y Th2 desbalanceadas ${ }^{37,41}$ y deficiencia relativa de linfocitos $\mathrm{CD} 4+^{42}$, y la suplementación con leptina normaliza la capacidad proliferativa de sus linfocitos CD4+ y CD45RA y así como los niveles circulantes de citoquinas Th $1^{42}$.

La concentración de leptina circulante correlaciona directamente con la actividad clínica de lupus eritematoso sistémico, esclerosis múltiple, tiroiditis postparto y enfermedad de Behçet ${ }^{43-46}$. Por otro lado, los ratones $o b / o b$ y $d b / d b$ son resistentes a enfermedades autoinmunes experimentales, tales como encefalomielitis, artritis, colitis, nefritis nefrotóxica y hepatitis ${ }^{40,47,48}$, sugiriendo 
que la leptina participa en la patogenia de algunas enfermedades autoinmunes.

Es frecuente que sujetos obesos desarrollen un estado inflamatorio crónico de bajo grado asociado a infiltración macrofágica del tejido adiposo. Esta inflamación adipocitaria podría determinar niveles aumentados de las citoquinas proinflamatorias TNF- $\alpha$, IL- $1 \beta$, IL- $6^{49}$, posiblemente contribuyendo al desarrollo de resistencia insulínica.

Para determinar si la leptina participa en la inflamación adipocitaria asociada a la obesidad, Gutiérrez y Hast generaron ratones con deficiencia selectiva de LepR en células hematopoyéticas. Contrariamente a lo esperado, la ausencia de LepR en células inmunes no determinó menor reclutamiento de macrófagos en el tejido adiposo, ni mayor resistencia insulínica en ratones alimentados con una dieta rica en grasa ${ }^{50}$, por lo que la relevancia de la leptina en la inflamación del tejido adiposo aún permanece incierta.

\section{B. Fibrosis hepática}

Diversas investigaciones sugieren que la leptina posee actividad profibrogénica directa en el hígado. Los ratones $o b / o b$ presentan resistencia al desarrollo de fibrosis hepática en modelos experimentales de estatohepatitis no alcohólica, así como en respuesta al daño hepatotóxico inducido con tetracloruro de carbono $\left(\mathrm{CCI}_{4}\right)^{51}$. Similarmente, ratas Zucker, que son genéticamente deficientes de LepR y, por lo tanto, genética y metabólicamente similares a los ratones $d b / d b$, tienen menores niveles de colágeno I y fibrosis hepática en respuesta a tioacetamida que sus contrapartes normales ${ }^{52}$. La infusión de leptina en estos animales restablece su capacidad fibrogénica hepática ${ }^{53}$.

Inversamente, la infusión de leptina potencia los efectos hepatotóxicos agudos del $\mathrm{CCl}_{4}$ y la fibrogénesis en ratones normales ${ }^{54}$.

En humanos se ha encontrado que la leptina circulante es mayor en pacientes con esteatohepatitis no alcohólica y que correlacionaría con la severidad anatomopatológica del daño hepático ${ }^{51}$. Esta correlación, sin embargo, pudiera ser meramente circunstancial, dada la alta prevalencia de obesidad y resistencia insulínica en pacientes con esteatohepatitis no alcohólica, ambos factores vinculados a la progresión de esta enfermedad hepática. Sin embargo, en un estudio reciente se encontró que la concentración circulante de leptina normalizada por los niveles de adiponectina, una adipoquina antiinflamatoria, correlaciona positiva, independiente y significativamente con mayor inflamación en pacientes con hígado graso no alcohólico ${ }^{55}$.

Las células estrelladas son las principales secretoras de colágeno en el hígado ${ }^{56}$ y normalmente expresan LepRb en su superficie. La incubación de células estrelladas con leptina in vitro induce la expresión de los genes codificantes para colágeno tipo I y para actina de músculo liso ${ }^{52}$, sugiriendo que la activación de LepRb es suficiente para inducir una respuesta fibrogénica en estas células.

LepR también es expresado por hepatocitos, células de Kupffer y células endoteliales sinusoidales. Se ha reportado que la activación de LepRb en las células de Kupffer in vitro induce la liberación de mediadores paracrinos activadores de las células estrelladas, tales como TGF-1b y CTGF/ $\mathrm{CCN} 2^{57}$, lo cual podría promover la acumulación de matriz extracelular fibrótica en el hígado.

Es importante notar que la relevancia clínica de estas últimas observaciones experimentales permanece incierta ya que no existen aún estudios en humanos que las evalúen directamente.

\section{Metabolismo óseo}

Los ratones $o b / o b$ tienen una masa ósea aumentada en comparación con animales de igual peso corporal ${ }^{58}$, sugiriendo que la leptina ejerce efectos reguladores negativos sobre la masa ósea corporal total.

En efecto, experimentos en animales y en cultivos primarios de osteoblastos y osteoclastos han mostrado que la leptina inhibe la formación de hueso a través de la reducción de la actividad osteoblástica, sin afectar la actividad osteoclástica ${ }^{58}$.

Notablemente, los osteoblastos no expresan LepR. Karsenty y cols. han trabajado en esta paradoja, mostrando que la acciones óseas de la leptina son indirectas e involucran la activación de LepRb el SNC, determinando una menor síntesis de serotonina en los núcleos del rafe del tronco cerebral y una mayor activación de la rama simpática del sistema nervioso autónomo ${ }^{59}$. De hecho, una actividad simpática aumentada se ha asociado con menores niveles de síntesis de hueso, secundarios a la activación de receptores adrenérgicos $\beta 2$ en osteoblastos $^{60}$. Consistentemente, pacientes con uso crónico de inhibidores de la recaptación de serotonina tienen mayor riesgo de fracturas ${ }^{61}$, sugiriendo que desbalances en la serotonina cerebral 
pudieran asociarse con enfermedades del hueso en seres humanos.

No obstante lo anterior, pacientes con anorexia nervosa hipoleptinémicas tienen una masa ósea disminuida en relación a personas de la misma edad y con masa corporal normal ${ }^{62}$. Esta discordancia con las observaciones anteriores (que la deficiencia de leptina favorece la ganancia de hueso) podría ser causada por el fenotipo complejo de esta enfermedad humana, en la cual además de modificaciones en la leptina circulante existen grandes mayores en el balance crónico de macro y micronutrientes así como de otros sistemas neuroendocrinos que pudieran anular el efecto protector de la hipoleptinemia sobre la pérdida de masa ósea.

\section{Reproducción}

Las mujeres jóvenes enflaquecidas tienen retraso puberal y las adultas con reducciones importantes de peso corporal desarrollan amenorrea secundaria, sugiriendo que el tejido adiposo genera señales que regulan la fisiología reproductiva femenina.

Este factor podría ser la leptina ${ }^{13}$.

Pacientes amenorreicas con anorexia nervosa ${ }^{63}$ o lipodistrofia generalizada presentan niveles muy bajos de leptina ${ }^{6}$ y los niveles de leptina correlacionan directamente con la presencia o ausencia de ciclos menstruales en mujeres enflaquecidas ${ }^{64}$.

A nivel experimental, las hembras $o b / o b$ tienen niveles muy reducidos de hormonas sexuales, no ovulan, no tienen ciclos estrales y permanecen en estado prepuberal indefinidamente ${ }^{65,66}$. La implantación de ovarios de hembras ob/ob en hembras normales restituye la capacidad ovulatoria de estos tejidos ${ }^{65}$, indicando que la infertilidad no es debida a defectos ováricos primarios, y la infusión de leptina en hembras $o b / o b$ restaura todos sus parámetros hormonales, así como sus ciclos estrales y fertilidad ${ }^{65}$.

En mujeres lipodistróficas la administración de leptina durante un año se ha asociado con niveles significativamente menores de testosterona circulante y mejoría de diversos parámetros de insulinorresistencia y síndrome de ovario poliquístico ${ }^{67}$.

Los mecanismos subyacentes a la acción de leptina sobre la fertilidad femenina son sólo parcialmente conocidos y dependen de la acción de esta adipoquina en el SNC. A nivel hipotalámico la liberación de la hormona liberadora de gonadotro- pinas $(\mathrm{GnRH})$ es inhibida por NPY (neuropéptido $\mathrm{Y}$ ), una hormona central en el control del apetito dependiente de leptina ${ }^{12}$. En efecto, la activación de LepRb en núcleos hipotalámicos inhibe la liberación de NPY, promoviendo la secreción de GnRH y gonadotropinas ${ }^{68}$.

El rol de leptina en la función reproductiva masculina ha sido menos explorado. Los machos ob/ob tienen fertilidad reducida y testículos y vesículas seminales de menor tamaño ${ }^{66}$. Esto animales presentan, además, diversas anormalidades histológicas testiculares, tales como menor volumen de los túbulos seminíferos, mayor proporción de células germinales en proceso de muerte celular por apoptosis ${ }^{69}$, niveles alterados de gonadotropinas e hipogonadismo manifiesto ${ }^{66}$. Se ha reportado que LepR es expresado por las células de Leydig en testículos de ratón ${ }^{70} \mathrm{y}$ en humanos ${ }^{71} \mathrm{y}$ aunque el tratamiento con leptina revierte la infertilidad en ratones $o b / o b$ macho $^{72}$ no se conoce la relevancia fisiológica o patológica de estas observaciones en seres humanos. Sólo se ha descrito una relación inversa, aunque poco potente, entre los niveles de expresión inmunohistoquímica de LepR testicular y los niveles de testosterona circulante en hombres ${ }^{71}$.

\section{E. Diabetes mellitus tipo 1}

La proyección clínica posiblemente más relevante de la leptina tiene que ver con su capacidad para tratar los trastornos metabólicos de la diabetes mellitus tipo $1(\mathrm{DM} 1)^{73}$. Resultados muy recientes muestran que esta hormona previene la hiperglicemia, lipólisis aumentada, cetoacidosis y muerte causada por deficiencia de insulina en modelos animales de $\mathrm{DM} 1^{74-76}$.

En el experimento original, reportado por Unger y cols., en University of Texas Southwestern Medical Center (Dallas, Estados Unidos de Norteamérica), la sobreexpresión de leptina por medio de transducción con adenovirus recombinante en ratones NOD (non-obese diabetic), un modelo murino de DM1, redujo la glicemia de $534 \pm 199$ $\mathrm{mg} / \mathrm{dL}$ a $77 \pm 67 \mathrm{mg} / \mathrm{dL}$, previno totalmente la acumulación de cuerpos cetónicos y normalizó los niveles de glucagón, aun en ausencia absoluta de insulina ${ }^{75}$. Similarmente, la infusión continua de leptina exógena en este modelo experimental mostró convincentemente que la monoterapia con leptina es suficiente para prevenir las alteraciones metabólicas y la muerte en ratones con $\mathrm{DM}{ }^{77}$. 
Los mecanismos subyacentes a estos efectos no están completamente dilucidados, sin embargo, parecen depender de la activación de LepRb en núcleos del SNC. En efecto, la administración intraventrículo-cerebral de leptina en ratones diabéticos con deficiencia absoluta de insulina replica todos los efectos benéficos de la infusión sistémica de esta adipoquina ${ }^{78}$.

A nivel metabólico, el tratamiento con leptina reduce en forma importante los niveles exagerados de glucagón observados en DM1, la expresión de enzimas gluconeogénicas y posiblemente la secreción aumentada de glucosa hepática ${ }^{75,77,78}$; sugiriendo que la leptina previene tanto la hiperglicemia como la cetoacidosis diabética a través de la supresión de la secreción de glucagón.

Estudios realizados en ratones con supresión genética del receptor de glucagón (RG) apoyan la hipótesis anterior ${ }^{79}$. De hecho, la reposición selectiva de RG en el hígado de estos animales restituye su sensibilidad a los desórdenes metabólicos derivados de la ausencia de insulina ${ }^{80}$.

Estas observaciones han hecho que Unger y Cherrington planteen que la DM1 es fundamentalmente la consecuencia metabólica de una actividad aumentada del sistema glucagón antes que del déficit primario de acción insulínica ${ }^{73}$ (Figura 3 ).

Aún no existen datos clínicos en pacientes que permitan extender estas ideas a la fisiopatología humana; sin embargo, está en curso el primer ensayo clínico de infusión de leptina en pacientes con DM1 (ClinicalTrial.gov, código NCT01268644).

\section{F. Diabetes mellitus tipo 2}

Recientemente se ha reportado un modelo animal de DM2, las ratas UCD-T2DM, que desarrollan obesidad poligénica de aparición adulta, resistencia insulínica y diabetes mellitus. Para estudiar la eficacia de leptina como posible tratamiento para DM2, se aplicó leptina por vía subcutánea a estos animales y se los comparó con animales tratados con solución salina ${ }^{81}$. Las ratas UCD-T2DM que recibieron leptina disminuyeron su peso corporal y glicemia y presentaron mejoría en sus parámetros de sensibilidad insulínica ${ }^{81}$. Sin embargo, la administración de leptina exógena en este trabajo determinó valores plasmáticos muy elevados ( 5 veces el valor previo al tratamiento), dificultando la interpretación de los resultados y su aplicabilidad clínica, ya que niveles suprafisiológicos de leptina pudieran asociarse a efectos

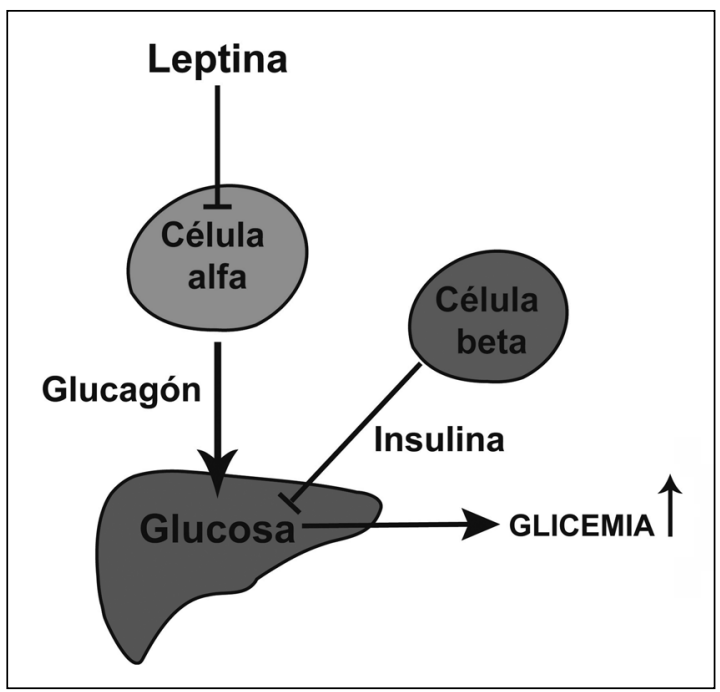

Figura 3. La leptina reduce la secreción hepática de glucosa por medio de la reducción de la secreción de glucagón por las células alfa de los islotes pancreáticos. La acción supresora de la leptina sobre el glucagón pancreático puede ser la consecuencia de la estimulación directa de LepR en las células alfa o de la acción de la leptina en el SNC, ya que la inyección intracerebroventricular de esta hormona en ratones genera el mismo resultado que su infusión sistémica (ver el texto principal para mayores detalles y referencias)

secundarios indeseables, tales como fibrosis hepática acelerada o desregulación inmune en sujetos predispuestos.

Agradecimientos: Los autores agradecen a Fondecyt (proyectos código 11100168, V.A.C. y 1120586, J.L.S.), Conicyt - Programa de Atracción e Inserción en la Academia (proyecto código 79100018, V.A.C.) y a la Dirección de Investigación de la Escuela de Medicina de la Pontificia Universidad Católica de Chile, Fondo de apoyo a la realización de tesis de postgrado (D.G.) por el apoyo financiero para la realización de este trabajo.

\section{Referencias}

1. Friedman JM. A tale of two hormones. Nat Med 2010; 16 (10): 1100-6

2. Ingalls AM, Dickie MM, Snell GD. Obese, a new mutation in the house mouse. J Hered 1950; 41 (12): 317-8.

3. Zhang Y, Proenca R, Maffei M, Barone M, Leopold L, Friedman JM. Positional cloning of the mouse obese 
gene and its human homologue. Nature 1994; 372 (6505): 425-32.

4. Montague CT, Farooqi IS, Whitehead JP, Soos MA, Rau $\mathrm{H}$, Wareham NJ, et al. Congenital leptin deficiency is associated with severe early-onset obesity in humans. Nature 1997; 387 (6636): 903-8.

5. Farooqi IS, Jebb SA, Langmack G, Lawrence E, Cheetham $\mathrm{CH}$, Prentice AM, et al. Effects of recombinant leptin therapy in a child with congenital leptin deficiency. N Engl J Med 1999; 341 (12): 879-84.

6. Oral EA, Simha V, Ruiz E, Andewelt A, Premkumar A, Snell P, et al. Leptin-replacement therapy for lipodystrophy. N Engl J Med 2002; 346 (8): 570-8.

7. Pelleymounter MA, Cullen MJ, Baker MB, Hecht R, Winters D, Boone T, et al. Effects of the obese gene product on body weight regulation in ob/ob mice. Science 1995; 269 (5223): 540-3.

8. Heymsfield SB, Greenberg AS, Fujioka K, Dixon RM, Kushner R, Hunt T, et al. Recombinant leptin for weight loss in obese and lean adults: a randomized, controlled, dose-escalation trial. JAMA 1999; 282 (16): 1568-75.

9. Ravussin E, Smith SR, Mitchell JA, Shringarpure R, Shan $\mathrm{K}$, Maier $\mathrm{H}$, et al. Enhanced weight loss with pramlintide/metreleptin: an integrated neurohormonal approach to obesity pharmacotherapy. Obesity (Silver Spring) 2009; 17 (9): 1736-43.

10. Hukshorn CJ, Saris WH, Westerterp-Plantenga MS, Farid AR, Smith FJ, Campfield LA. Weekly subcutaneous pegylated recombinant native human leptin (PEG-OB) administration in obese men. J Clin Endocrinol Metab 2000; 85 (11): 4003-9.

11. Myers MG Jr, Leibel RL, Seeley RJ, Schwartz MW. Obesity and leptin resistance: distinguishing cause from effect. Trends Endocrinol Metab 2010; 21(11): 643-51.

12. Santos MJ. [Leptin-melanocortin system, body weight regulation and obesity]. Rev Med Chile 2009; 137 (9): 1225-34.

13. Friedman JM, Halaas JL. Leptin and the regulation of body weight in mammals. Nature 1998; 395 (6704): 763-70.

14. Madej T, Boguski MS, Bryant SH. Threading analysis suggests that the obese gene product may be a helical cytokine. FEBS Lett 1995; 373 (1): 13-8.

15. Tartaglia LA, Dembski M, Weng X, Deng N, Culpepper J, Devos R, et al. Identification and expression cloning of a leptin receptor, OB-R. Cell 1995; 83 (7): 1263-71.

16. Masuzaki H, Ogawa Y, Sagawa N, Hosoda K, Matsumoto $\mathrm{T}$, Mise $\mathrm{H}$, et al. Nonadipose tissue production of leptin: leptin as a novel placenta-derived hormone in humans. Nat Med 1997; 3 (9): 1029-33.

17. Bado A, Levasseur S, Attoub S, Kermorgant S, Laigneau
JP, Bortoluzzi MN, et al. The stomach is a source of leptin. Nature 1998; 394 (6695): 790-3.

18. Lee GH, Proenca R, Montez JM, Carroll KM, Darvishzadeh JG, Lee JI, et al. Abnormal splicing of the leptin receptor in diabetic mice. Nature 1996; 379 (6566): 632 5.

19. Tartaglia LA. The leptin receptor. J Biol Chem 1997; 272 (10): 6093-6.

20. Fruhbeck $\mathrm{G}$. Intracellular signalling pathways activated by leptin. Biochem J 2006; 393 (Pt 1): 7-20.

21. Chen H, Charlat O, Tartaglia LA, Woolf EA, Weng X, Ellis SJ, et al. Evidence that the diabetes gene encodes the leptin receptor: identification of a mutation in the leptin receptor gene in $\mathrm{db} / \mathrm{db}$ mice. Cell 1996; 84 (3): 491-5.

22. Elmquist JK, Bjorbaek C, Ahima RS, Flier JS, Saper CB. Distributions of leptin receptor mRNA isoforms in the rat brain. J Comp Neurol 1998; 395 (4): 535-47.

23. Cohen P, Zhao C, Cai X, Montez JM, Rohani SC, Feinstein $\mathrm{P}$, et al. Selective deletion of leptin receptor in neurons leads to obesity. J Clin Invest 2001; 108 (8): 1113-21.

24. Hileman SM, Pierroz DD, Masuzaki H, Bjorbaek C, ElHaschimi K, Banks WA, et al. Characterizaton of short isoforms of the leptin receptor in rat cerebral microvessels and of brain uptake of leptin in mouse models of obesity. Endocrinology 2002; 143 (3): 775-83.

25. Ogier V, Ziegler O, Mejean L, Nicolas JP, StrickerKrongrad A. Obesity is associated with decreasing levels of the circulating soluble leptin receptor in humans. Int J Obes Relat Metab Disord 2002; 26 (4): 496-503.

26. Sun Q, van Dam RM, Meigs JB, Franco OH, Mantzoros CS, Hu FB. Leptin and soluble leptin receptor levels in plasma and risk of type 2 diabetes in U.S. women: a prospective study. Diabetes 2010; 59 (3): 611-8.

27. Botella Carretero JI, Lledin Barbancho MD, Valero González MA, Varela Dacosta C. [Leptin: physiological and clinical role]. An Med Interna 2001; 18 (3): 152-60.

28. Bruning JC, Gautam D, Burks DJ, Gillette J, Schubert $\mathrm{M}$, Orban PC, et al. Role of brain insulin receptor in control of body weight and reproduction. Science 2000; 289 (5487): 2122-5.

29. Ozata M, Ozdemir IC, Licinio J. Human leptin deficiency caused by a missense mutation: multiple endocrine defects, decreased sympathetic tone, and immune system dysfunction indicate new targets for leptin action, greater central than peripheral resistance to the effects of leptin, and spontaneous correction of leptin-mediated defects. J Clin Endocrinol Metab 1999; 84 (10): 3686-95.

30. Matarese G, Alviggi C, Sanna V, Howard JK, Lord GM, Carravetta $\mathrm{C}$, et al. Increased leptin levels in serum and 
peritoneal fluid of patients with pelvic endometriosis. J Clin Endocrinol Metab 2000; 85 (7): 2483-7.

31. Torpy DJ, Bornstein SR, Chrousos GP. Leptin and interleukin-6 in sepsis. Horm Metab Res 1998; 30 (12): 726-9.

32. Janik JE, Curti BD, Considine RV, Rager HC, Powers GC, Alvord WG, et al. Interleukin 1 alpha increases serum leptin concentrations in humans. J Clin Endocrinol Metab 1997; 82 (9): 3084-6.

33. Matarese G, Moschos S, Mantzoros CS. Leptin in immunology. J Immunol 2005; 174 (6): 3137-42.

34. Zarkesh-Esfahani H, Pockley G, Metcalfe RA, Bidlingmaier $\mathrm{M}$, Wu Z, Ajami A, et al. High-dose leptin activates human leukocytes via receptor expression on monocytes. J Immunol 2001; 167 (8): 4593-9.

35. Faggioni R, Feingold KR, Grunfeld C. Leptin regulation of the immune response and the immunodeficiency of malnutrition. FASEB J 2001; 15 (14): 2565-71.

36. Caldefie-Chezet F, Poulin A, Vasson MP. Leptin regulates functional capacities of polymorphonuclear neutrophils. Free Radic Res 2003; 37 (8): 809-14.

37. Lord GM, Matarese G, Howard JK, Baker RJ, Bloom SR, Lechler RI. Leptin modulates the T-cell immune response and reverses starvation-induced immunosuppression. Nature 1998; 394 (6696): 897-901.

38. Tian Z, Sun R, Wei H, Gao B. Impaired natural killer (NK) cell activity in leptin receptor deficient mice: leptin as a critical regulator in NK cell development and activation. Biochem Biophys Res Commun 2002; 298 (3): 297-302.

39. Howard JK, Lord GM, Matarese G, Vendetti S, Ghatei MA, Ritter MA, et al. Leptin protects mice from starvation-induced lymphoid atrophy and increases thymic cellularity in ob/ob mice. J Clin Invest 1999; 104 (8): 1051-9.

40. La Cava A, Matarese G. The weight of leptin in immunity. Nat Rev Immunol 2004; 4 (5): 371-9.

41. Flier JS. Lowered leptin slims immune response. Nat Med 1998; 4 (10): 1124-5.

42. Farooqi IS, Matarese G, Lord GM, Keogh JM, Lawrence E, Agwu C, et al. Beneficial effects of leptin on obesity, T cell hyporesponsiveness, and neuroendocrine/metabolic dysfunction of human congenital leptin deficiency. J Clin Invest 2002; 110 (8): 1093-103.

43. García-González A, González-López L, Valera-González IC, Cardona-Muñoz EG, Salazar-Paramo M, GonzálezOrtiz M, et al. Serum leptin levels in women with systemic lupus erythematosus. Rheumatol Int 2002; 22 (4): 138-41.

44. Batocchi AP, Rotondi M, Caggiula M, Frisullo G, Odoardi F, Nociti V, et al. Leptin as a marker of multiple sclerosis activity in patients treated with interferon-beta. J Neuroimmunol 2003; 139 (1-2): 150-4.

45. Mazziotti G, Parkes AB, Lage M, Premawardhana LD, Casanueva FF, Lazarus JH. High leptin levels in women developing postpartum thyroiditis. Clin Endocrinol (Oxf) 2004; 60 (2): 208-13.

46. Evereklioglu C, Inaloz HS, Kirtak N, Doganay S, Bulbul $\mathrm{M}$, Ozerol E, et al. Serum leptin concentration is increased in patients with Behcet's syndrome and is correlated with disease activity. Br J Dermatol 2002; 147 (2): 331-6.

47. Chandra RK. Cell-mediated immunity in genetically obese C57BL/6J ob/ob) mice. Am J Clin Nutr 1980; 33 (1): 13-6.

48. Mandel MA, Mahmoud AA. Impairment of cell-mediated immunity in mutation diabetic mice $(\mathrm{db} / \mathrm{db})$. J Immunol 1978; 120 (4): 1375-7.

49. Xu H, Barnes GT, Yang Q, Tan G, Yang D, Chou CJ, et al. Chronic inflammation in fat plays a crucial role in the development of obesity-related insulin resistance. J Clin Invest 2003; 112 (12): 1821-30.

50. Gutiérrez DA, Hasty AH. Haematopoietic leptin receptor deficiency does not affect macrophage accumulation in adipose tissue or systemic insulin sensitivity. J Endocrinol 2012; 212 (3): 343-51.

51. Saxena NK, Ikeda K, Rockey DC, Friedman SL, Anania FA. Leptin in hepatic fibrosis: evidence for increased collagen production in stellate cells and lean littermates of ob/ob mice. Hepatology 2002; 35 (4): 762-71.

52. Ikejima K, Takei Y, Honda H, Hirose M, Yoshikawa M, Zhang YJ, et al. Leptin receptor-mediated signaling regulates hepatic fibrogenesis and remodeling of extracellular matrix in the rat. Gastroenterology 2002; 122 (5): 1399410.

53. Leclercq IA, Farrell GC, Schriemer R, Robertson GR. Leptin is essential for the hepatic fibrogenic response to chronic liver injury. J Hepatol 2002; 37 (2): 206-13.

54. Ikejima K, Honda $\mathrm{H}$, Yoshikawa M, Hirose M, Kitamura $\mathrm{T}$, Takei Y, et al. Leptin augments inflammatory and profibrogenic responses in the murine liver induced by hepatotoxic chemicals. Hepatology 2001; 34 (2): 288-97.

55. Lemoine M, Ratziu V, Kim M, Maachi M, Wendum D, Paye F, et al. Serum adipokine levels predictive of liver injury in non-alcoholic fatty liver disease. Liver International 2009; 29 (9): 1431-8.

56. Friedman SL. Molecular regulation of hepatic fibrosis, an integrated cellular response to tissue injury. J Biol Chem 2000; 275 (4): 2247-50.

57. Wang J, Leclercq I, Brymora JM, Xu N, RamezaniMoghadam M, London RM, et al. Kupffer cells mediate leptin-induced liver fibrosis. Gastroenterology 2009; 137 (2): 713-23. 
Leptina en salud y enfermedad - D. Goldenberg et al

58. Ducy P, Amling M, Takeda S, Priemel M, Schilling AF, Beil FT, et al. Leptin inhibits bone formation through a hypothalamic relay: a central control of bone mass. Cell 2000; 100 (2): 197-207.

59. Yadav VK, Oury F, Suda N, Liu ZW, Gao XB, Confavreux $\mathrm{C}$, et al. A serotonin-dependent mechanism explains the leptin regulation of bone mass, appetite, and energy expenditure. Cell 2009; 138 (5): 976-89.

60. Takeda S, Elefteriou F, Levasseur R, Liu X, Zhao L, Parker $\mathrm{KL}$, et al. Leptin regulates bone formation via the sympathetic nervous system. Cell 2002; 111 (3): 305-17.

61. Richards JB, Papaioannou A, Adachi JD, Joseph L, Whitson HE, Prior JC, et al. Effect of selective serotonin reuptake inhibitors on the risk of fracture. Arch Intern Med 2007; 167 (2): 188-94.

62. Grinspoon S, Miller K, Coyle C, Krempin J, Armstrong C, Pitts S, et al. Severity of osteopenia in estrogen-deficient women with anorexia nervosa and hypothalamic amenorrhea. J Clin Endocrinol Metab 1999; 84 (6): 2049-55.

63. Casanueva FF, Dieguez C, Popovic V, Peino R, Considine RV, Caro JF. Serum immunoreactive leptin concentrations in patients with anorexia nervosa before and after partial weight recovery. Biochem Mol Med 1997; 60 (2): 116-20.

64. Di Carlo C, Tommaselli GA, De Filippo E, Pisano G, Nasti A, Bifulco G, et al. Menstrual status and serum leptin levels in anorectic and in menstruating women with low body mass indexes. Fertil Steril 2002; 78 (2): 376-82.

65. Chehab FF, Lim ME, Lu R. Correction of the sterility defect in homozygous obese female mice by treatment with the human recombinant leptin. Nat Genet 1996; 12 (3): 318-20.

66. Swerdloff RS, Batt RA, Bray GA. Reproductive hormonal function in the genetically obese (ob/ob) mouse. Endocrinology 1976; 98 (6): 1359-64.

67. Lungu AO, Zadeh ES, Goodling A, Cochran E, Gorden P. Insulin resistance is a sufficient basis for hyperandrogenism in lipodystrophic women with polycystic ovarian syndrome. J Clin Endocrinol Metab 2012; 97 (2): 563-7.

68. Stephens TW, Basinski M, Bristow PK, Bue-Valleskey JM, Burgett SG, Craft L, et al. The role of neuropeptide Y in the antiobesity action of the obese gene product. Nature 1995; 377 (6549): 530-2.

69. Bhat GK, Sea TL, Olatinwo MO, Simorangkir D, Ford $\mathrm{GD}$, Ford BD, et al. Influence of a Leptin Deficiency on Testicular Morphology, Germ Cell Apoptosis, and Expression Levels of Apoptosis-Related Genes in the Mouse. J Androl 2006; 27 (2): 302-10.

70. Cioff JA, Shafer AW, Zupancic TJ, Smith-Gbur J, Mikhail A, Platika D, et al. Novel B219/OB receptor isoforms: possible role of leptin in hematopoiesis and reproduction. Nat Med 1996; 2 (5): 585-9.

71. Ishikawa T, Fujioka H, Ishimura T, Takenaka A, Fujisawa M. Expression of leptin and leptin receptor in the testis of fertile and infertile patients. Andrologia 2007; 39 (1): 22-7.

72. Mounzih K, Lu R, Chehab FF. Leptin Treatment Rescues the Sterility of Genetically Obese ob/ob Males. Endocrinology 1997; 138 (3): 1190-3.

73. Unger RH, Cherrington AD. Glucagonocentric restructuring of diabetes: a pathophysiologic and therapeutic makeover. J Clin Invest 2012; 122 (1): 4-12.

74. Koch L, Wunderlich FT, Seibler J, Konner AC, Hampel $\mathrm{B}$, Irlenbusch $\mathrm{S}$, et al. Central insulin action regulates peripheral glucose and fat metabolism in mice. J Clin Invest 2008; 118 (6): 2132-47.

75. Yu X, Park BH, Wang MY, Wang ZV, Unger RH. Making insulin-deficient type 1 diabetic rodents thrive without insulin. Proc Natl Acad Sci U S A 2008; 105 (37): 14070 5.

76. Chinookoswong N, Wang JL, Shi ZQ. Leptin restores euglycemia and normalizes glucose turnover in insulindeficient diabetes in the rat. Diabetes 1999; 48 (7): 1487 92.

77. Wang MY, Chen L, Clark GO, Lee Y, Stevens RD, Ilkayeva OR, et al. Leptin therapy in insulin-deficient type I diabetes. Proc Natl Acad Sci U S A 2010; 107 (11): 4813-9.

78. Fujikawa T, Chuang JC, Sakata I, Ramadori G, Coppari R. Leptin therapy improves insulin-deficient type 1 diabetes by CNS-dependent mechanisms in mice. Proc Natl Acad Sci U S A 2010; 107 (40): 17391-6.

79. Lee Y, Wang MY, Du XQ, Charron MJ, Unger RH. Glucagon receptor knockout prevents insulin-deficient type 1 diabetes in mice. Diabetes 2011; 60 (2): 391-7.

80. Lee Y, Berglund ED, Wang MY, Fu X, Yu X, Charron MJ, et al. Metabolic manifestations of insulin deficiency do not occur without glucagon action. Proc Natl Acad Sci U S A 2012; 109 (37): 14972-6.

81. Cummings BP, Bettaieb A, Graham JL, Stanhope KL, Dill R, Morton GJ, et al. Subcutaneous administration of leptin normalizes fasting plasma glucose in obese type 2 diabetic UCD-T2DM rats. Proc Natl Acad Sci U S A 2011; 108 (35): 14670-5. 